\title{
Extension of soil thermal conductivity models to frozen meats with low and high fat content
}

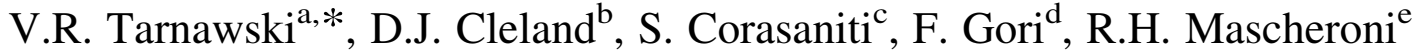 \\ ${ }^{a}$ Division of Engineering, Saint Mary's University 923 Robie St, Halifax, Canada B3H 3C3 \\ ${ }^{\mathrm{b}}$ Institute of Technology and Engineering, Massey University Palmerston, New Zealand \\ 'Department of Mechanical Engineering, University of Rome 'Tor Vergata' 00133 Roma, Italy \\ ${ }^{\mathrm{d}}$ Department of Mechanical Engineering, University of Rome 'Tor Vergata' 00133 Roma, Italy \\ ${ }^{\mathrm{e}}$ CIDCA and Departamento Ingeniería Química, Facultad de Ingeniería, UNLP_47 y 116 (1900) La Plata, Argentina.
}

Received 24 November 2003; received in revised form 12 January 2005; accepted 31 January 2005

Available online 2 April 2005

\begin{abstract}
Thermal conductivity models of frozen soils were analyzed and compared with similar models developed for frozen foods. In total, eight thermal conductivity models and 54 model versions were tested against experimental data of 13 meat products in the temperature range from 0 to $-40{ }^{\circ} \mathrm{C}$. The model by deVries, with water + ice $(w i)$ as the continuous phase, showed overall the best predictions. The use of wi leads generally to improved predictions in comparison to ice; water as the continuous phase is beneficial only to deVries model, mostly from -1 to $-20{ }^{\circ} \mathrm{C}$; fat is advantageous only to meats with high fat content. The results of this work suggest that the more sophisticated way of estimating the thermal conductivity for a disperse phase in the deVries model might be more appropriate than the use of basic multi-phase models (geometric mean, parallel, and series). Overall, relatively small differences in predictions were observed between the best model versions by deVries, Levy, Mascheroni, Maxwell or Gori as applied to frozen meats with low content of fat. These differences could also be generated by uncertainty in meat composition, temperature dependence of thermal conductivity of ice, measurement errors, and limitation of predictive models.
\end{abstract}

(C) 2005 Elsevier Ltd and IIR. All rights reserved.

\section{Modèles de la conductivité thermique du sol appliqués aux viandes surgelées à faible et à forte teneur en matière grasse}

Mots clés : Produit congelé ; Modélisation ; Conductivité thermique ; Expérimentation ; Comparaison ; Sol

\section{Introduction}

* Corresponding author.

E-mail address: vlodek.tarnawski@smu.ca

(V.R. Tarnawski).
Soils and foods are both heterogeneous porous media and share some similar features as well as a number of differences. Soils are mixtures of inorganic loose particles of 


\begin{tabular}{|c|c|c|c|}
\hline \multicolumn{4}{|c|}{ Nomenclature } \\
\hline$a, b, c$ & axes of ellipsoidal food components & $w i_{\|}$ & water + ice $=$continuous phase; $\lambda$ evaluated by \\
\hline$a_{0}, a_{1}, a$ & constants in Eqs. (20) and (21) & & $\|$ model (Eq. (1)) \\
\hline$F$ & alternative function for $\theta_{\mathrm{d}}$ used in Levy's model & $\mathrm{wi}_{\sum}$ & water + ice $=$ continuous phase; $\lambda$ evaluated by \\
\hline f & fat as continuous phase & & $\sum$ (Eq. (2)) \\
\hline$f-d_{G M M}$ & $\begin{array}{l}\text { fat as continuous phase; } \lambda \text { of dispersed phase } \\
\text { (d) evaluated by GMM (Eq. (3a)) }\end{array}$ & Greek & \\
\hline$f-d_{\|}$ & $\begin{array}{l}\text { fat as continuous phase; } \lambda \text { of dispersed phase } \\
\text { (d) evaluated by II (Eq. (1)) }\end{array}$ & & $\begin{array}{l}\text { variable in Eqs. (13) and (14) } \\
\text { volume fraction }\end{array}$ \\
\hline$f-d_{\sum}$ & fat as continuous phase; $\lambda$ of dispersed phase & & $\begin{array}{l}\text { thermal conductivity }\left(\mathrm{W} / \mathrm{m}{ }^{\circ} \mathrm{C}\right) \\
\text { density }\end{array}$ \\
\hline $\mathrm{fib}_{\mathrm{GMM}}$ & $\begin{array}{l}\lambda_{\text {fiber }} \text { (models by Mascheroni)-evaluated by } \\
\text { GMM (Eq. (3a)) }\end{array}$ & & $\begin{array}{l}\lambda_{\mathrm{d}} / \lambda_{\text {con }} \\
\text { variable in Eqs. (7) and (8) }\end{array}$ \\
\hline $\mathrm{fib}_{\|}$ & $\begin{array}{l}\lambda_{\text {fiber }} \text { (models by Mascheroni)-evaluated by } \| \\
\text { (Eq. (1)) }\end{array}$ & $\begin{array}{l}\xi \\
\text { Subscrip }\end{array}$ & variable in Eqs. (15) and (16) \\
\hline $\mathrm{fib}_{\sum}$ & $\begin{array}{l}\lambda_{\text {fiber }} \text { (models by Mascheroni)-evaluated by } \sum \\
\text { (Eq. (2)) }\end{array}$ & a & $\begin{array}{l}\text { air } \\
\text { ash/mineral }\end{array}$ \\
\hline$g$ & shape factor & $\mathrm{b}$ & bulk \\
\hline$k$ & weighting factor & bw & bound water \\
\hline i & ice as continuous phase & car & carbohydrate \\
\hline $\mathrm{i}-\mathrm{d}_{\mathrm{GMM}}$ & $\begin{array}{l}\text { ice as continuous phase; } \lambda \text { of dispersed phase } \\
\text { (d) evaluated by GMM (Eq. (3a)) }\end{array}$ & $\begin{array}{l}\text { con } \\
d\end{array}$ & $\begin{array}{l}\text { continuous phase } \\
\text { dispersed phase }\end{array}$ \\
\hline$i-d_{\|}$ & $\begin{array}{l}\text { ice as continuous phase; } \lambda \text { of dispersed phase } \\
\text { (d) evaluated by } \| \text { (Eq. (1)) }\end{array}$ & $\begin{array}{l}\exp \\
\mathrm{f}\end{array}$ & $\begin{array}{l}\text { experimental } \\
\text { initial freezing point }\end{array}$ \\
\hline $\mathrm{i}-\mathrm{d}_{\sum}$ & $\begin{array}{l}\text { ice as continuous phase; } \lambda \text { of dispersed phase } \\
\text { (d) evaluated by } \sum \text { (Eq. (2)) }\end{array}$ & $\begin{array}{l}\text { fat } \\
\text { fib }\end{array}$ & $\begin{array}{l}\text { fat } \\
\text { meat-fibre }\end{array}$ \\
\hline$M$ & mass fraction & GMM & geometric mean model \\
\hline$N$ & the number of solid components & ice & ice \\
\hline$n$ & number of phases, data records, etc. & $\mathrm{j}$ & food component number \\
\hline$p$ & $\begin{array}{l}\text { ellipsoid shape value (equatorial diameter/- } \\
\text { distance between ellipsoid poles) }\end{array}$ & prot & $\begin{array}{l}\text { proteins } \\
\text { solids }\end{array}$ \\
\hline RMSE & root mean square error & un & unfrozen water \\
\hline$s_{\mathrm{GMM}}$ & solids $\lambda$ evaluated by GMM (Eq. (3a)) & $\mathrm{w}$ & water \\
\hline$T$ & temperature $\left({ }^{\circ} \mathrm{C}\right)$ & wi & water + ice \\
\hline $\mathrm{w}$ & water as continuous phase & $\|$ & heat flow $\|$ to fibers of meat \\
\hline & water + ice as continuous phase & $\perp$ & heat flow $\perp$ to fibers of meat \\
\hline $\mathrm{wi}_{\mathrm{GMM}}$ & $\begin{array}{l}\text { water }+ \text { ice }=\text { continuous phase; } \lambda \text { evaluated by } \\
\text { GMM (Eq. (3a)) }\end{array}$ & & \\
\hline
\end{tabular}

various sizes and shapes, organic matter, water, and air. The total volume fraction of water and air is known as the soil porosity. Foods are heterogeneous capillary-porous colloidal materials composed of numerous solid constituents, such as carbohydrates, fats, proteins, vitamins and minerals, plus water; air voids can also be present. In the food literature, porosity generally refers to the air component only. Porosity of soils (relative volume of air plus water) typically varies from 30 to $60 \%$ while for foods moisture content generally varies from 15 to $90 \%$. The water, containing dissolved substances, is a major component in both soils and foods and its transition from liquid into ice is the greatest factor influencing change in thermal properties with temperature $(T)$. In soils, water exists in gravitational, capillary, and hygroscopic (bound) forms, while foods contain only capillary and bound water. Foods are generally saturated with water, while soils experience great variation of water content, from dryness to a field capacity (volumetric water content at saturation minus the gravitational water). A large part of the water, in both soils and foods, freezes rapidly between 0 and $-5^{\circ} \mathrm{C}$, therefore, very sparse thermal conductivity $(\lambda)$ data is available in this $T$ range. Excluding water, ice and air, the thermal conductivity of other food components are similar, while in soils the thermal conductivity of quartz is large compared with other mineralogical constituents. Soil composition is usually given by volumetric fractions, while for foods mass fraction is most commonly employed.

Analysis, design and simulation of food freezing and 


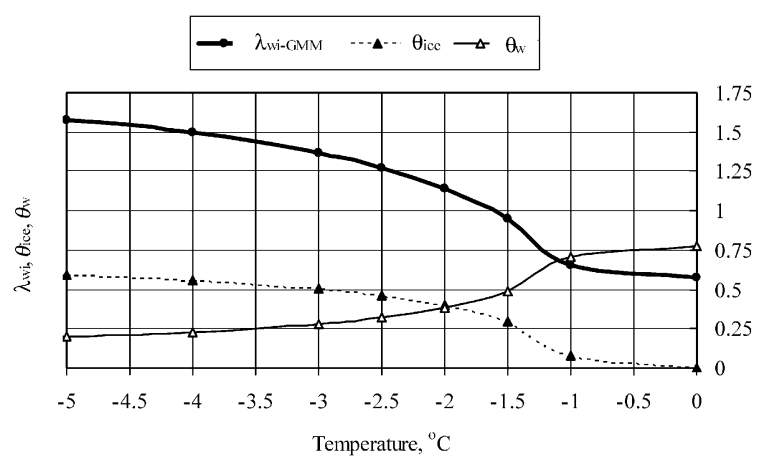

Fig. 1. Volumetric content of ice $\left(\theta_{\text {ice }}\right)$, water $\left(\theta_{\mathrm{w}}\right)$ and $\lambda_{\text {wi-GMM }}$ vs. $T$ for leg-muscle-parallel.

storage process demands reliable and easily accessible thermal property data across a wide range of temperatures, particularly below the freezing point. The $\lambda$ of frozen foods is a key property to the above applications. In general, experimental determination of $\lambda$ is difficult, time and labour expensive, and error prone. Therefore, estimation of $\lambda$ from predictive models, based on food composition, is often employed. A similar modelling approach has also been used in soils. Apart from the basic series and parallel models and combination of these, the most commonly quoted thermal conductivity models in the frozen food literature are the Maxwell-Eucken equation [1], Levy's modification to the Maxwell-Eucken equation [2] and the effective medium theory (EMT) [3]. Mascheroni et al. [4] proposed a model for frozen meat which was verified against a limited set of experimental data only. Pham [5] found Levy's model the most accurate of seven models (the model by Mascheroni was not considered) tested for meats with low and high fat content. Models developed for soil systems that could be extended to frozen foods, include: the weighted average by deVries [6], a cubic cell by Gori [7], self-consistent approximation by Sundberg [8], and geometric mean by Lichtenecker [9]. The existing literature shows lack of clear evidence of the application of frozen soil models to frozen foods.

\section{Scope of the paper}

The purpose of this paper is to evaluate the application of frozen soil models to food systems and compare them with the best frozen food models under assumption that thermal conductivity of food components is not dependent on $T$. The $\lambda$ models developed from the original Maxwell relationship (Maxwell-Eucken, Levy, deVries) require component information regarding the continuous (con) and dispersed phases $(d)$. Commonly, the dominant volumetric fraction of food component (usually water or ice) is assumed as the continuous medium. This assumption, however, may not be entirely true, as at the beginning of freezing, the unfrozen water content can still be a dominant phase, i.e. larger than newly formed ice fraction (Fig. 1). In addition, changing the continuous phase from water into ice leads to a discontinuity problem, i.e. a step change of $\lambda$ as the $\lambda_{\text {ice }}>4 \lambda_{\mathrm{w}}$. These problems, might be eliminated if water and ice are treated as one continuous phase (wi) whose lumped conductivity $\lambda_{\text {wi }}$ changes smoothly over the entire freezing $T$ range, i.e. from $\lambda_{\mathrm{w}}$ (unfrozen foods) to $\lambda_{\text {ice }}$ (fully frozen foods)-Fig. 1 . The lumped $\lambda_{\text {wi }}$ and $\lambda_{\mathrm{d}}$ can be evaluated by one of the simple multi-phase models such as geometric mean, parallel and series. Therefore, another objective of this paper is to examine the influence of the continuous phase selection (water, ice, water+ice, fat) on the effective thermal conductivity of frozen foods.

\section{Review of predictive models for foods and soils}

\subsection{Basic multi-phase models}

The majority of the above models require additional data regarding the lumped thermal conductivity of the continuous and dispersed phases. This information is usually obtained by applying parallel, series, or geometric-mean models. The parallel model (II) assumes parallel configuration of the system components in the direction of heat flow:

$\lambda=\frac{\sum_{1}^{n} \theta_{j} \lambda_{j}}{\sum_{1}^{n} \theta_{j}}$

The series model $\left(\sum\right)$ assumes series configuration of the system components in the direction of heat flow:

$\lambda=\frac{\sum_{1}^{n} \theta_{j}}{\sum_{1}^{n} \frac{\theta_{j}}{\lambda_{j}}}$

The geometric-mean model (GMM), proposed by Lichtenecker [9], is not based on any physical concepts, but it is commonly used due to its simplicity.

$\lambda=\prod_{1}^{n} \lambda_{j}^{\theta_{j}}$

The results produced by the GMM model are intermediate between the $\|$ and $\sum$ model. Good predictive results of this model, for frozen saturated soils, were reported by Johansen [10] who used the following form of this model:

$\lambda=\lambda_{\mathrm{s}}^{\theta_{\mathrm{s}}} \lambda_{\text {ice }}^{\theta_{\text {ice }}} \lambda_{\mathrm{un}}^{\theta_{\mathrm{un}}}$

This model structure can be easily applied for frozen foods because the evaluation of the lumped conductivity of solid components, $\lambda_{s}$, excludes the ice and other water fractions.

In general, the thermal conductivity models can be subdivided into two groups; the first group considering only 


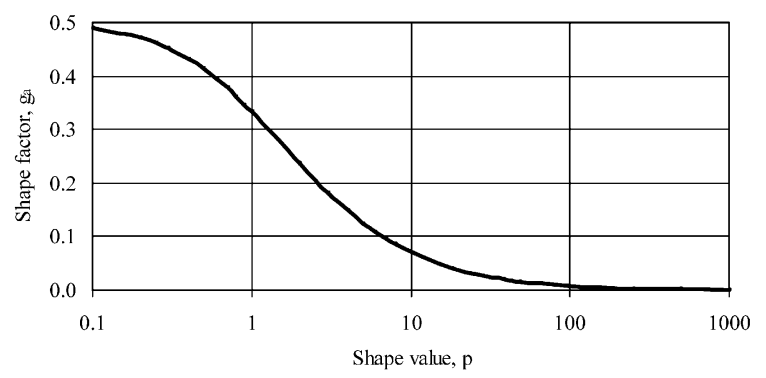

Fig. 2. Shape factor $g_{a}$ as a function of the ellipsoid shape value $p$.

two phases, continuous and dispersed one (e.g. MaxwellEucken, Levy), and the second one with more than two phases (solid, liquid, gaseous).

\subsection{Two-phases models}

\subsubsection{Maxwell-Eucken}

Originally, this model was developed for predicting the electrical conductivity of non-interacting homogeneous spheres (dispersed phase) in a homogeneous continuous phase. The of this binary system $(\operatorname{con}+d)$ was given by:

$\lambda=\lambda_{\operatorname{con}} \frac{1+\frac{2}{\delta}+2 \theta_{\mathrm{d}}\left(1-\frac{1}{\delta}\right)}{1+\frac{2}{\delta}-\theta_{\mathrm{d}}\left(1-\frac{1}{\delta}\right)}$

where $\delta=\lambda_{\mathrm{d}} / \lambda_{\text {con }}$. When applied to frozen foods, this model gives different $\lambda$ values depending on which components are chosen to be a part of the continuous and dispersed phases and how the lumped $\lambda$ of each phase is estimated. Eq. (4) can be converted into a weighted average form in terms of the continuous and dispersed phases [11]:

$\lambda=\frac{k_{\text {con }} \theta_{\text {con }} \lambda_{\text {con }}+k_{\mathrm{d}} \theta_{\mathrm{d}} \lambda_{\mathrm{d}}}{k_{\mathrm{con}} \theta_{\text {con }}+k_{\mathrm{d}} \theta_{\mathrm{d}}}$

$k_{\mathrm{d}}=\frac{1}{3} \sum_{x=a b c}\left[1+g_{x}(\delta-1)\right]^{-1}=\frac{3}{\delta+2}$

where, $k_{\mathrm{con}}=1, g_{\mathrm{x}}$ is a shape factor for each dimension of particles in the dispersed phase.

For solid spheres $(a=b=c), g_{x}=1 / 3$. The $\lambda_{\mathrm{d}}$ can be

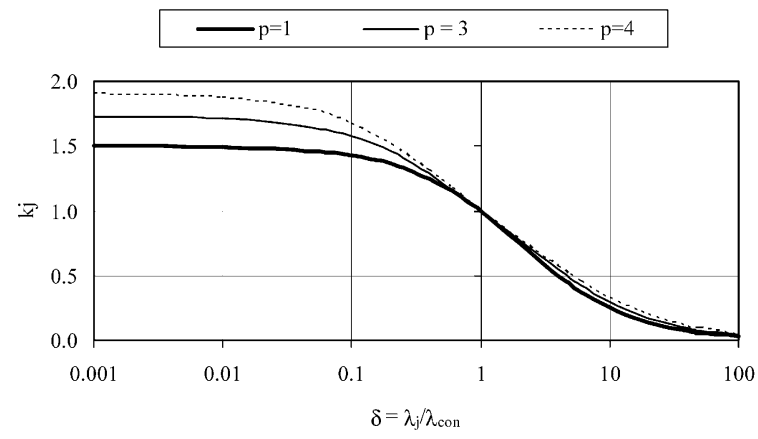

Fig. 3. Variation of $k_{j}$ factor vs. $\lambda_{j} \lambda_{\text {con }}$ for $p=1-3-4$. evaluated by one of basic multiphase models such as, $\sum$, $\|$, or GMM.

\subsubsection{Levy modification to Maxwell-Eucken model}

Levy [2] modified the Maxwell-Eucken model (Eq. (4)) by replacing the volume fraction of a dispersed phase, $\theta_{\mathrm{d}}$, by a function $F$ so that the predictions are independent of the designation of phases as continuous or dispersed:

$F=\frac{1}{\sigma}-\frac{1}{2}+\theta_{\mathrm{d}}-\frac{1}{2} \sqrt{\left(\frac{2}{\sigma}-1+2 \theta_{\mathrm{d}}\right)^{2}-8 \frac{\theta_{\mathrm{d}}}{\sigma}}$

$\sigma=\frac{(\delta-1)^{2}}{(\delta+1)^{2}+0.5 \delta}$

\subsection{Multi-phases models}

\subsection{1. deVries model}

Fricke [12] was the first to consider extension of the Maxwell's model to homogeneous ellipsoidal particles. Later on, the same idea was used by de Vries [6] for predicting $\lambda$ of moist soils; i.e. a spherical shape of solid grains was replaced with rotated oblate ellipsoids dispersed in the continuous medium (water, air).

$\lambda=\frac{k_{\mathrm{con}} \theta_{\mathrm{con}} \lambda_{\mathrm{con}}+\sum_{1}^{N} k_{j} \theta_{j} \lambda_{j}}{k_{\mathrm{con}} \theta_{\mathrm{con}}+\sum_{1}^{N} k_{j} \theta_{j}}$

where $N$ is the number of solid components; each solid grain of component $(j)$ has the same weighting factor $k_{j}$ and the same thermal conductivity $\lambda_{j}$.

If the axes $(a, b, c)$ of ellipsoidal granules are oriented randomly, $k_{j}$ can be obtained from

$k_{j}=\frac{1}{3} \sum_{x=a b c}\left[1+\left(\frac{\lambda_{j}}{\lambda_{\text {con }}}-1\right) g_{x}\right]^{-1}=\frac{(\nabla T)_{j}}{(\nabla T)_{\text {con }}}$

According to de Vries [6] $k_{j}$ represents the ratio of the average $T$ gradient in a solid constituent ( $j$ ) to the average $T$ gradient in the continuous phase. In practice, $k_{j}$ is an adjustment factor improving $\lambda$ predictions. Eq. (10) converts to unity $\left(k_{\mathrm{con}}=1\right)$ if the $j$ constituent is assumed as the continuous phase $\left(\lambda_{j}=\lambda_{\text {con }}\right)$. For the dispersed phase, the $k_{j}$ depends on $\lambda_{j} / \lambda_{\text {con }}$ and a solid particle ellipsoidal shape factor for each of three axes $\left(g_{x=a, b, c}\right)$. The shape factors $g_{a}$, $g_{b}$, and $g_{c}$ depend on ratios of the ellipsoid axes $a, b$, and $c$. The sum of $g_{a}, g_{b}$, and $g_{c}$ is unity. For rotated ellipsoids ( $a=$ $b), g_{a}=g_{b}, g_{c}=1-2 g_{a}$. The $g_{a}$ values can be estimated from equations given by Carslaw and Jaeger [13]: oblate ellipsoids: $p=a / c>1$

$g_{a}=\frac{p^{2}}{2 \sqrt{\left(p^{2}-1\right)^{3}}}\left[\frac{\pi}{2}-\arctan \left(\frac{1}{\sqrt{p^{2}-1}}\right)-\frac{\sqrt{p^{2}-1}}{p^{2}}\right]$

prolate ellipsoids: $p=a / c<1$ 


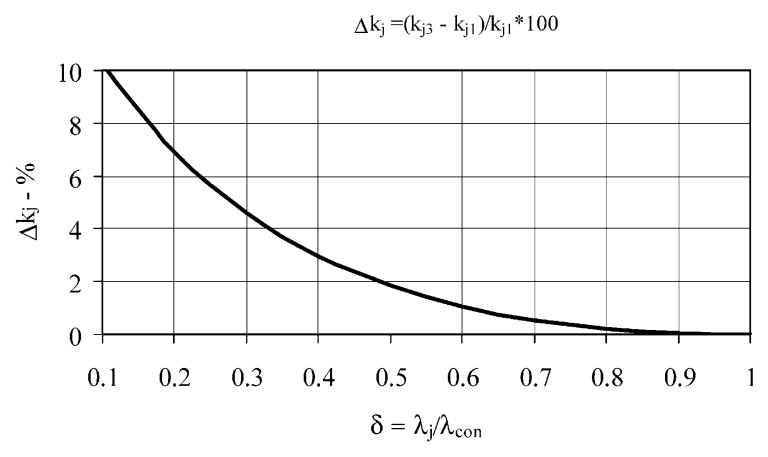

Fig. 4. Relative $\Delta k_{j}$ change with $\delta$ (oblate ellipsoid, $p=3$ wrt. sphere, $p=1$ ).

$g_{a}=0.5\left[\frac{1}{1-p^{2}}-\frac{p^{2}}{2 \sqrt{\left(1-p^{2}\right)^{3}}} \ln \left(\frac{1+\sqrt{1-p^{2}}}{1-\sqrt{1-p^{2}}}\right)\right]$

Variation of the shape factor $g_{a}$ as a function of a shape value $p$ is shown on Fig. 2. Examples of the $k_{j}$ values, for rotated ellipsoids ( $p=3$ and 4$)$ and for a sphere $(p=1)$ vs. the ratio $\lambda_{j} / \lambda_{\text {con }}$, are shown in Fig. 3. The model is simple to use when applied to saturated porous media. It requires, however, knowledge regarding the shape of solid grains, which can vary from oblate to prolate ellipsoids, thus including also a sphere. For a system made of spherical solids $\left(a=b=c ; g_{a}=g_{b}=g_{c}=1 / 3\right)$, Eq. (9) reduces to a form very similar in appearance to the Maxwell's model (Eq. (5)). When both models (Eqs. (5) and (9)) are to be applied to frozen foods, it is important to be aware of some hidden disparities. First, it is more likely that solid components resemble the shape of oblate ellipsoids $(p>1)$ rather then perfect spheres $(p=1)$. Secondly, Eq. (5) treats the dispersed components as a single phase, while Eq. (9) handles each dispersed component individually. The spherical weighting factor $k_{\mathrm{d}}$ depends on $\delta$ only, while $k_{j}$ depends on the $\delta$ and also on the shape value $p$ of each individual component of the dispersed phase. For foods, $\delta$ varies approximately from 0.1 to 0.3 and the use of $p$ values for oblate ellipsoids rather than sphere (e.g. $p=3$ ) leads to relative change in $k_{j}$ ranging from 5 to $10 \%$ (Fig. 4). Furthermore, in Eq. (5), $\lambda_{\mathrm{d}}$ is evaluated by one of simple multi-phase models (Eqs. (1)-(3)), while individual $\lambda_{j}$ values are used in Eq. (9).

\subsubsection{Gori's model}

The model assumes a cubic cell of lumped solids surrounded by another cubic cell composed of unfrozen water and ice as the continuous medium. For foods, the model was converted from a three phase system (solids, water, ice) into a two phase system consisting of food solids as a dispersed phase and wi as the continuous phase. The first model considers the horizontal parallel isotherms (hpiwi) in the cubic cell. $\frac{1}{\lambda}=\frac{\beta-1}{\lambda_{\mathrm{wi}} \beta}+\frac{\beta}{\lambda_{\mathrm{wi}}\left[\beta^{2}-1\right]+\lambda_{\mathrm{s}}}$

The second model takes into account a vertical parallel heat flux (vphf-wi) in the cubic cell.

$\lambda=\frac{1}{\frac{\beta(\beta-1)}{\lambda_{\mathrm{wi}}}+\frac{\beta}{\lambda_{\mathrm{s}}}}+\lambda_{\mathrm{wi}} \frac{\beta^{2}-1}{\beta^{2}}, \quad \beta=\sqrt[3]{\frac{1}{1-\theta_{\mathrm{w}}-\theta_{a}}}$

\subsubsection{Mascheroni's model}

The model was developed for solid frozen meats with the following assumptions: water in fibres was treated as a randomly dispersed phase in a continuous matrix of the remaining food tissue; partially dehydrated fibres were surrounded by ice; at $T>T_{\mathrm{f}}$ all the water was within the fibre; and, at $T<T_{\mathrm{f}}$ ice was being formed in the extra cellular space at expense of water in the fibres. One model option considers that heat flow is perpendicular $(\perp)$ to the meat fibres:

$\lambda_{\perp}=\frac{\lambda_{\text {ice }} \lambda_{\text {fib }}\left(1-\xi_{\text {ice }}\right)}{\xi_{\text {ice }} \lambda_{\text {fib }}+\lambda_{\text {ice }}\left(1-\xi_{\text {ice }}\right)}+\lambda_{\text {ice }} \xi_{\text {ice }}$

The other model option is based on assumption that heat flow is parallel $(\|)$ to the meat fibers:

$$
\begin{aligned}
\lambda_{\|}= & \lambda_{\text {ice }} \xi_{\text {ice }}+\left(1-\xi_{\text {ice }}\right) \\
& \times\left\{\lambda_{\text {ice }} \xi_{\text {ice }}^{2}+\lambda_{\text {fib }}\left(1-\xi_{\text {ice }}\right)^{2}+\frac{4 \xi_{\text {ice }}\left(1-\xi_{\text {ice }}\right)}{\frac{1}{\lambda_{\text {fib }}}+\frac{1}{\lambda_{i}}}\right\}
\end{aligned}
$$

where $\xi_{\text {ice }}=1-\sqrt{1-\theta_{\text {ice }}}$. Originally, the thermal conductivity of meat fibers, $\lambda_{\text {fib }}$, was evaluated by the MaxwellEucken model using the fiber component composition. In this paper, however, the $\lambda_{\text {fib }}$ is modeled by simple multiphase models (GMM, $\|, \sum$ ).

\subsubsection{Self-consistent approximation (SCA)-effective medium theory (EMT) model}

The SCA model was originally developed by Bruggeman [14] for predicting $\lambda$ of a mixture of isotropic materials. Later on, the same expression was utilized by Landeauer [15] as the effective medium theory (EMT) model and used for evaluating the electrical resistance of binary metallic mixtures. Then, the model was adapted to predicting the $\lambda$ of vegetable foods [3].

$\sum_{1}^{n} \theta_{j} \frac{\lambda_{j}-\lambda}{\lambda_{j}+2 \lambda}=0$

Sundberg [8] used the same model, a slightly different form, for predicting $\lambda$ of soils.

$\lambda=\frac{1}{3} \frac{1}{\sum_{1}^{3} \frac{\theta_{i}}{\lambda_{j}+2 \lambda}}$

In both forms (Eqs. (17) and (18)), $\theta_{j}$ and $\lambda_{j}$ are the volume fraction and thermal conductivity of the $j$ component, 
Table 1

Mass composition of the meats under investigation

\begin{tabular}{lllllll}
\hline Food & Code \# & $M_{\mathrm{w}}$ & $M_{\text {prot }}$ & $M_{\text {fat }}$ & $M_{\text {ash }}$ & $M_{\text {car }}$ \\
\hline Leg muscle $\|$ & 1 & 0.736 & 0.199 & 0.047 & 0.011 & 0.007 \\
Leg muscle minced & 2 & 0.739 & 0.186 & 0.045 & 0.01 & 0.02 \\
Hearts & 3 & 0.698 & 0.15 & 0.123 & 0.012 & 0.017 \\
Hearts minced & 4 & 0.688 & 0.149 & 0.139 & 0.01 & 0.014 \\
Livers & 5 & 0.689 & 0.194 & 0.062 & 0.015 & 0.04 \\
Livers minced & 6 & 0.677 & 0.208 & 0.037 & 0.02 & 0.058 \\
Brains & 7 & 0.79 & 0.103 & 0.076 & 0.014 & 0.017 \\
Kidneys & 8 & 0.799 & 0.145 & 0.031 & 0.013 & 0.012 \\
Thymus & 9 & 0.7919 & 0.143 & 0.064 & 0.001 & 0.0001 \\
Thymus minced & 10 & 0.7589 & 0.135 & 0.092 & 0.014 & 0.0001 \\
Leg muscle $\perp$ & 11 & 0.725 & 0.193 & 0.072 & 0.009 & 0.001 \\
Fat & 12 & 0.1329 & 0.032 & 0.834 & 0.001 & 0.0001 \\
Fat minced & 13 & 0.1109 & 0.029 & 0.858 & 0.002 & 0.0001 \\
\hline
\end{tabular}

Note. The $M_{\text {car }}$ have been causing a calculation problem in the model by Mascheroni, therefore, instead of 0 a very small number 0.0001 was assigned, at expense of $M_{\mathrm{w}}$.

respectively. If the system is composed of more than two phases, both equations have an implicit form and must be solved by an iterative method. In fact, frozen foods and soils are examples of three-phase systems, i.e. consisting of unfrozen water, ice and solids.

$\lambda_{1}=\lambda_{\mathrm{w}} \quad \theta_{1}=\theta_{\mathrm{un}} \quad \lambda_{2}=\lambda_{\text {ice }} \quad \theta_{2}=\theta_{\text {ice }}$

$\lambda_{3}=\lambda_{\mathrm{s}} \quad \theta_{3}=1-\theta_{\mathrm{w}}-\theta_{a}$

\subsubsection{Modified resistor series model}

Cleland [16] proposed a modified resistor series model applied to calculating $\lambda$ of frozen foods.

$$
\frac{\theta_{\mathrm{w}}+\theta_{\mathrm{bw}}+\theta_{\mathrm{s}}}{\lambda}=\frac{\theta_{\mathrm{w}}+\theta_{\mathrm{bw}}}{\lambda_{\mathrm{w}}}+\frac{\theta_{\mathrm{s}}}{\sum_{j=1}^{n} \frac{\theta_{j}}{\theta_{\mathrm{s}}} \lambda_{j}}
$$

\section{Experimental data and model testing}

The reviewed models were tested against the experimental $\lambda$ data in the $T$ range 0 to $-40{ }^{\circ} \mathrm{C}$ for 13 meats with low and high fat content (Table 1) published by Pham and Willix [17]. The food component mass fractions in this data set do not always sum exactly to 1 . When the mass fractions were smaller than 1, then the remainder was arbitrarily assigned to carbohydrates. If there was a surplus, then all the component mass fractions were proportionally reduced. None of the products contained significant air voids. The experimental data covers only a few points for each product, which restricts comparison with the predictive models. Therefore, instead of the real experimental data, the following empirical equations, developed by Pham and Willix [17] were used.

$T>T_{\mathrm{f}} \quad \lambda=\lambda_{\mathrm{f}}+a_{0}\left(T-T_{\mathrm{f}}\right)$
$T<T_{\mathrm{f}} \quad \lambda=\lambda_{\mathrm{f}}+a_{1}\left(T-T_{\mathrm{f}}\right)+a_{2}\left(\frac{1}{T}-\frac{1}{T_{\mathrm{f}}}\right)$

The fitted parameters are given in Table 2. The thermal conductivity and density data for the food product components used to make predictions was assumed to be $T$ independent and their values (Table 3 ) were given by Choi and Okos [18].

The mass fraction of unfrozen water (including bound water) was estimated from:

$M_{\mathrm{un}}=M_{\mathrm{w}}-M_{\text {ice }}$

In the frozen range, the mass fraction of ice content was estimated from the widely used empirical relationship, developed by Schwartzberg [19], which is based on Raoult's law of dilute solutions and the Claussius-Clapeyron relationship:

$M_{\text {ice }}=\left(M_{\mathrm{w}}-M_{\mathrm{bw}}\right)\left(1-\frac{T_{\mathrm{f}}}{T}\right)$

The freezing point and bound water mass fractions were based on the data and relationships given by Pham and Willix [17]:

$T_{\mathrm{f}}=-0.9{ }^{\circ} \mathrm{C} \quad M_{\mathrm{bw}}=0.4 M_{\mathrm{prot}}$

The mass fractions of all components were converted to volumetric fractions.

$\theta_{j}=M_{j} \frac{\rho_{b}(T)}{\rho_{j}}$

The bulk density of frozen food $\rho_{b}(T)$ for $T<T_{\mathrm{f}}$ can be obtained from:

$\rho_{b}(T)=\frac{1}{\sum_{j=1}^{n} \frac{M_{j}}{\rho_{j}}}$

Table 4 gives the volumetric composition of the unfrozen 

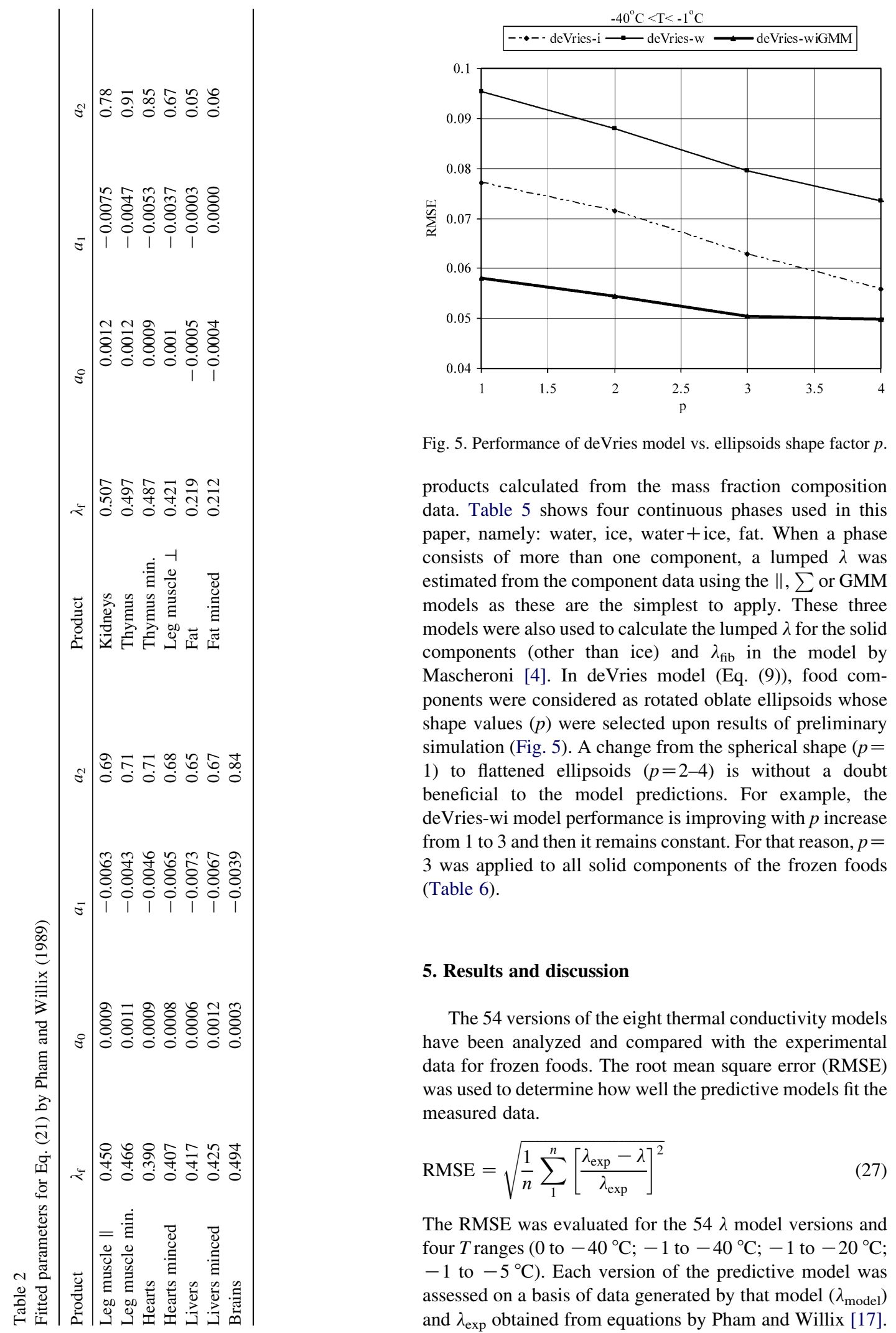

Fig. 5. Performance of deVries model vs. ellipsoids shape factor $p$.

products calculated from the mass fraction composition data. Table 5 shows four continuous phases used in this paper, namely: water, ice, water + ice, fat. When a phase consists of more than one component, a lumped $\lambda$ was estimated from the component data using the $\|, \sum$ or GMM models as these are the simplest to apply. These three models were also used to calculate the lumped $\lambda$ for the solid components (other than ice) and $\lambda_{\text {fib }}$ in the model by Mascheroni [4]. In deVries model (Eq. (9)), food components were considered as rotated oblate ellipsoids whose shape values $(p)$ were selected upon results of preliminary simulation (Fig. 5). A change from the spherical shape ( $p=$ 1) to flattened ellipsoids $(p=2-4)$ is without a doubt beneficial to the model predictions. For example, the deVries-wi model performance is improving with $p$ increase from 1 to 3 and then it remains constant. For that reason, $p=$ 3 was applied to all solid components of the frozen foods (Table 6).

\section{Results and discussion}

The 54 versions of the eight thermal conductivity models have been analyzed and compared with the experimental data for frozen foods. The root mean square error (RMSE) was used to determine how well the predictive models fit the measured data.

$\operatorname{RMSE}=\sqrt{\frac{1}{n} \sum_{1}^{n}\left[\frac{\lambda_{\exp }-\lambda}{\lambda_{\exp }}\right]^{2}}$

The RMSE was evaluated for the $54 \lambda$ model versions and four $T$ ranges $\left(0\right.$ to $-40{ }^{\circ} \mathrm{C} ;-1$ to $-40{ }^{\circ} \mathrm{C} ;-1$ to $-20{ }^{\circ} \mathrm{C}$; -1 to $-5^{\circ} \mathrm{C}$ ). Each version of the predictive model was assessed on a basis of data generated by that model $\left(\lambda_{\text {model }}\right)$ and $\lambda_{\exp }$ obtained from equations by Pham and Willix [17]. 
Table 3

Thermal conductivity and density of food components by Choi and Okos (1986)

\begin{tabular}{lcccccc}
\hline Component & Water & Ice & Protein & Fat & Ash & Carbohydrate \\
\hline$\lambda\left(\mathrm{W} / \mathrm{m} \mathrm{K}^{3}\right.$ & 0.56 & 2.21 & 0.20 & 0.18 & 0.33 & 0.20 \\
$\rho\left(\mathrm{kg} / \mathrm{m}^{3}\right)$ & 1000 & 917 & 1380 & 930 & 2424 & 1600 \\
\hline
\end{tabular}

Table 4

Volume composition of the unfrozen meats under investigation

\begin{tabular}{|c|c|c|c|c|c|c|c|}
\hline Food & Code \# & $\rho_{\mathrm{b}}$ & $\theta_{\mathrm{w}}$ & $\theta_{\text {prot }}$ & $\theta_{\text {fat }}$ & $\theta_{\text {ash }}$ & $\theta_{\text {car }}$ \\
\hline Leg muscle ॥ & 1 & 1055.5 & 0.779 & 0.158 & 0.054 & 0.005 & 0.005 \\
\hline Leg muscle minced & 2 & 1056.8 & 0.783 & 0.148 & 0.051 & 0.004 & 0.013 \\
\hline Hearts & 3 & 1040.3 & 0.728 & 0.117 & 0.138 & 0.005 & 0.011 \\
\hline Hearts minced & 4 & 1036.2 & 0.715 & 0.116 & 0.156 & 0.004 & 0.009 \\
\hline Livers & 5 & 1069.5 & 0.739 & 0.156 & 0.072 & 0.007 & 0.027 \\
\hline Livers minced & 6 & 1087.2 & 0.738 & 0.170 & 0.043 & 0.009 & 0.039 \\
\hline Brains & 7 & 1032.8 & 0.818 & 0.080 & 0.085 & 0.006 & 0.011 \\
\hline Kidneys & 8 & 1045.3 & 0.838 & 0.114 & 0.035 & 0.006 & 0.008 \\
\hline Thymus & 9 & 1029.5 & 0.818 & 0.111 & 0.071 & 0.000 & 0.000 \\
\hline Thymus minced & 10 & 1033.3 & 0.786 & 0.105 & 0.103 & 0.006 & 0.000 \\
\hline Leg muscle $\perp$ & 11 & 1047.9 & 0.762 & 0.152 & 0.082 & 0.004 & 0.001 \\
\hline Fat & 12 & 944.4 & 0.126 & 0.023 & 0.851 & 0.000 & 0.000 \\
\hline Fat minced & 13 & 942.6 & 0.105 & 0.021 & 0.874 & 0.001 & 0.000 \\
\hline
\end{tabular}

For $T$ range from -1 to $-5{ }^{\circ} \mathrm{C}$, the $T$ increment was $-0.5^{\circ} \mathrm{C}$, and $-2.5^{\circ} \mathrm{C}$, from -5 to $-40{ }^{\circ} \mathrm{C}$.

Tables 7-9 summarize the average RMSE for the best 10 model versions applied to 11 low fat meats (high water content), two high fat meats (low water content) and all 13 meats under investigation, respectively. As far as the $\lambda$ of lean meats (11 foods with high water content) is concerned, Masch- $\perp-f i b_{\|}$, deVries-wi $i_{G M M}$ and de Vries- $w$ model offer clearly the best predictions in $T$ range from -1 to $-5{ }^{\circ} \mathrm{C}$ and -1 to $-20^{\circ} \mathrm{C}$. Slightly less accurate predictions in these two $T$ ranges are given by Gori-vphf-wi and Maxwell$w i_{G M M}$ (Fig. 6). In the $T$ range from -1 to $-40{ }^{\circ} \mathrm{C}$ ), deVries-wi $i_{G M M}$ produces the best predictions followed by deVries- $i$ and then by a large group of models (Maxwell$w i_{G M M}$, Gori-vphf-wi, Masch-\|-fib ${ }_{G M M}$, Masch-\|-fib ${ }_{\sum}$, $E T M-s_{G M M}$, deVries-w), whose performance is more or less at the same level. The predictive performance of the Vries- $w$ and the Masch- $\perp-f i b_{\|}$model, however, is declining for $T$ ranging from -20 to $-40{ }^{\circ} \mathrm{C}$, i.e. when a large majority of water has been converted into the ice. The Masch-\|-fib $b_{G M M}$ and Maxwell-wi $i_{G M M}$ predict well in all three ranges of freezing $T$. The Levy-wi $i_{\|}$model performs

Table 5

Options for continuous and dispersed phases in frozen foods

\begin{tabular}{ll}
\hline Continuous phase (con) & Dispersed phase $(d)$ \\
\hline Ice $(i)$ & Food solid components + water \\
Water + ice $(w i)$ & Food solid components \\
Water $(w)$ & Food solid components + ice \\
Fat $(f)$ & Food solid components + ice + water \\
\hline
\end{tabular}

better than Levy-i-d $d_{G M M}$ and offers acceptable performance in $\Delta T$ range from -1 to $-20^{\circ} \mathrm{C}$ and -1 to $-40{ }^{\circ} \mathrm{C}(8$ and 9 th place, respectively), but it largely over-predicts the data near the freezing point $\left(-1\right.$ to $\left.-5{ }^{\circ} \mathrm{C}\right)$. The $E M T-s_{G M M}$ and Gori-vphf-wi models are simple in form and provide good $\lambda$ estimates for frozen and unfrozen foods. The model by Cleland [16] gives acceptable performance (10th place) in the full range of freezing $T$ only. With regard to high fat meats (two foods with very low water content), Levy-wi $i_{\|}$, deVries- $w$ and a wide variety of Levy model versions show the best predictions in $T$ range from -1 to $-5{ }^{\circ} \mathrm{C}$ and -1 to $-20{ }^{\circ} \mathrm{C}$. For the full range of freezing $T\left(-1\right.$ to $\left.-40{ }^{\circ} \mathrm{C}\right)$, Levy-f- $d_{\|}$, Levy-wi $i_{\|}$, deVries- $w$, and Levy-i- $d_{\|}$have the best predictions with very small differences between them. For

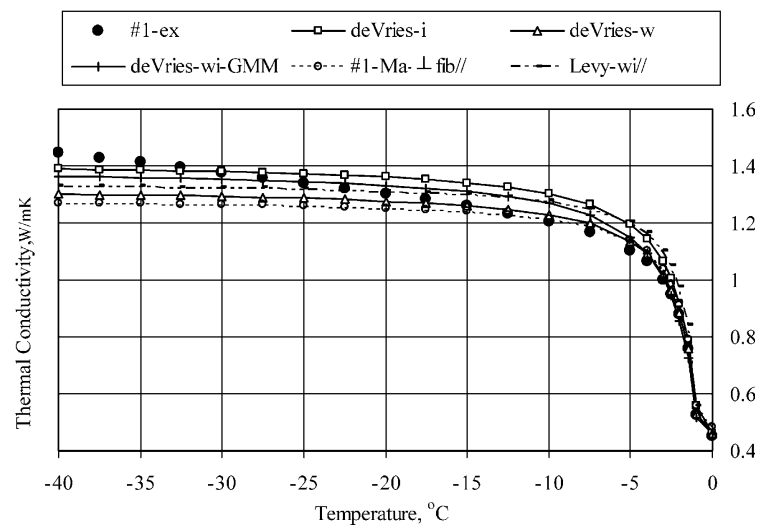

Fig. 6. The experimental $\lambda$ vs. predictive data (frozen lamb leg muscle parallel). 
Table 6

Shape factors for food components

\begin{tabular}{lllllll}
\hline Meat components & Water & Protein & Fat & Ash & Carb-hyd & Ice \\
\hline Shape value $(p)$ & 100 & 3 & 3 & 3 & 3 & 3 \\
Shape factor $(g)$ & 0.01 & 0.18 & 0.18 & 0.18 & 0.18 & 0.18 \\
\hline
\end{tabular}

Table 7

Best predictive models-low fat meats (1-11)

\begin{tabular}{|c|c|c|c|c|c|c|c|}
\hline $0{ }^{\circ} \mathrm{C}$ & RMSE & $\left\langle-1^{\circ} \mathrm{C} \ldots-5^{\circ} \mathrm{C}\right\rangle$ & RMSE & $\left\langle-1{ }^{\circ} \mathrm{C} \ldots-20^{\circ} \mathrm{C}\right\rangle$ & RMSE & $\left\langle-1^{\circ} \mathrm{C} \ldots-40^{\circ} \mathrm{C}\right\rangle$ & RMSE \\
\hline GeoMean-s $\mathrm{GMM}$ & 0.041 & Masch- $\perp-$ fib $_{\|}$ & 0.047 & Masch- $\perp-$ fib $_{\|}$ & 0.048 & deVries-wi ${ }_{\mathrm{GMM}}$ & 0.050 \\
\hline Levy-w & 0.046 & deVries-w & 0.048 & Gori-vphf-wi & 0.051 & deVries-i & 0.058 \\
\hline Levy-f-d $\mathrm{d}_{\mathrm{GMM}}$ & 0.046 & deVries-wi GMM $_{1}$ & 0.048 & deVries-wib $_{\mathrm{GMM}}$ & 0.047 & Maxwell-wi ${ }_{\mathrm{GMM}}$ & 0.058 \\
\hline EMT-s ${ }_{\mathrm{GMM}}$ & 0.047 & Maxwell-wi $\mathrm{GMM}_{\mathrm{GM}}$ & 0.052 & deVries-w & 0.052 & Gori-vphf-wi & 0.058 \\
\hline deVries-w & 0.048 & Masch- $\perp-$ fib $_{\mathrm{GMM}}$ & 0.057 & deVries-i & 0.060 & Masch-\|-fib ${ }_{\mathrm{GMM}}$ & 0.058 \\
\hline Gori-vphf-w & 0.048 & EMT-s $\mathrm{s}_{\mathrm{GMM}}$ & 0.058 & Masch-\|-fígM & 0.063 & Masch-\|-fib ${ }_{\sum}$ & 0.059 \\
\hline Maxwell-f-d $\mathrm{d}_{\mathrm{GMM}}$ & 0.050 & deVries-i & 0.059 & Maxwell-wi ${ }_{\mathrm{GMM}}$ & 0.064 & EMT-s $\mathrm{s}_{\mathrm{GMM}}$ & 0.061 \\
\hline deVries-f & 0.050 & Masch-\|-fib $b_{\mathrm{GMM}}$ & 0.061 & Levy-wi $\|$ & 0.064 & deVries-w & 0.061 \\
\hline Maxwell-w & 0.050 & Gori-vphf-wi & 0.061 & Masch-\|-fib ${ }_{\sum}$ & 0.066 & Levy-wi ${ }_{\|}$ & 0.070 \\
\hline Masch- $\perp-\mathrm{fib}_{\mathrm{GMM}}$ & 0.050 & Masch- $\|-f i b_{\sum}$ & 0.067 & EMT-s $\mathrm{s}_{\mathrm{GMM}}$ & 0.067 & Cleland & 0.071 \\
\hline
\end{tabular}

Table 8

Best predictive models - high fat meats (12-13)

\begin{tabular}{|c|c|c|c|c|c|c|c|}
\hline $0{ }^{\circ} \mathrm{C}$ & RMSE & $\left\langle-1{ }^{\circ} \mathrm{C} \ldots-5^{\circ} \mathrm{C}\right\rangle$ & RMSE & $\left\langle-1{ }^{\circ} \mathrm{C} \ldots-20^{\circ} \mathrm{C}\right\rangle$ & RMSE & $\left\langle-1^{\circ} \mathrm{C} \ldots-40^{\circ} \mathrm{C}\right\rangle$ & RMSE \\
\hline deVries-w & 0.003 & Levy-wi-d $\|_{\|}$ & 0.028 & Levy-wi-d ${ }_{\|}$ & 0.032 & Levy-f-d ${ }_{\|}$ & 0.031 \\
\hline deVries-f & 0.004 & deVries-w & 0.029 & de Vries-w & 0.032 & Levy-wi $\|$ & 0.034 \\
\hline Gori-vphf-w & 0.004 & Levy-f-d $\|$ & 0.033 & Levy-f-d ${ }_{\|}$ & 0.036 & deVries-w & 0.036 \\
\hline Levy-f-d $d_{\|}$ & 0.005 & Levy-wi ${ }_{\mathrm{GMM}}$ & 0.036 & Levy-i-d $d_{\|}$ & 0.039 & Levy-i-d ${ }_{\|}$ & 0.037 \\
\hline Maxwell-w & 0.006 & Maxwell-wi ${ }_{\sum}$ & 0.037 & Levy-wi ${ }_{\mathrm{GMM}}$ & 0.040 & deVries-f & 0.046 \\
\hline Gori-hpi-w & 0.008 & deVries-wi $_{\sum}$ & 0.038 & deVries-wi $_{\sum}$ & 0.047 & Levy-wi ${ }_{\mathrm{GMM}}$ & 0.046 \\
\hline Maxwell-f-d ${ }_{\|}$ & 0.012 & Levy-i-d $d_{\|}$ & 0.041 & Levy-i-d ${ }_{\mathrm{GMM}}$ & 0.049 & Levy-i $\mathrm{i}_{\mathrm{GMM}}$ & 0.053 \\
\hline Levy-w & 0.015 & Levy-i-d $\mathrm{d}_{\mathrm{GMM}}$ & 0.048 & Levy-f-d $\mathrm{GMM}_{\mathrm{M}}$ & 0.055 & deVries-wi $i_{\sum}$ & 0.060 \\
\hline Levy-f-d $\mathrm{d}_{\mathrm{GMM}}$ & 0.026 & Levy-f-d $\mathrm{d}_{\mathrm{GMM}}$ & 0.051 & Levy-i-d ${ }_{\sum}$ & 0.065 & Levy-f-d $\mathrm{d}_{\mathrm{GMM}}$ & 0.064 \\
\hline EMT-s ${ }_{\mathrm{GMM}}$ & 0.027 & deVries-f & 0.060 & Levy-wi $_{\sum}$ & 0.069 & Levy-i-d ${ }_{\sum}$ & 0.071 \\
\hline
\end{tabular}

all meats under investigation (11 lean +2 high fat), deVries$w i_{G M M}$, deVries- $w$ and Levy-wi $i_{\|}$exhibit the best performance in all $T$ ranges followed by Masch- $\perp-f i b_{\|}$, Gori-vphf-wi and $E T M-s_{G M M}$. The $\lambda$ of all unfrozen meat products $\left(0{ }^{\circ} \mathrm{C}\right)$ was predicted very well by a large group of models (Table 9).

\section{Conclusions and recommendations}

The analysis of computer simulation confirms that deVries-wi $i_{G M M}$ has overall the best predictions for frozen meats, while for unfrozen meats, a large majority of tested models show very accurate predictions. In general, the model by deVries has a good physical basis, requires relatively small calculation effort and has a potential for further improvement, particularly in the shape description of oblate ellipsoids. The results of this work suggest also that the more sophisticated way of estimating the $\lambda_{d}$ for a disperse phase in the deVries model might be more appropriate than the use of basic multi-phase models (GMM, II, $\sum$ ). This could also be a reason that in general the deVries model performed slightly better than the other models. It appears that the performance of predictive models, as applied to frozen foods, is strongly influenced by the choice of continuous phase. The use of wi has a sound physical basis as both water and ice exist practically in the full range of freezing $T$. The freezing process, particularly between -1 and $-5{ }^{\circ} \mathrm{C}$, is simulated better with the use of $w i$ than $i$ alone. For this reason, the choice of wi as the continuous phase leads to improved $\lambda$ predictions in comparison to ice $(i)$. In addition, it is also easier to assess the $\lambda_{\mathrm{d}}$ of the dispersed phase as the thermal conductivities of solid food components are more or less in the same range. The use of $w i$ in deVries model requires, however, additional calculations for $k_{j}$, which strongly depends on 
Table 9

Best predictive models-overall (1-13)

\begin{tabular}{|c|c|c|c|c|c|c|c|}
\hline $0{ }^{\circ} \mathrm{C}$ & RMSE & $\left\langle-1^{\circ} \mathrm{C} \ldots-5^{\circ} \mathrm{C}\right\rangle$ & RMSE & $\left\langle-1{ }^{\circ} \mathrm{C} \ldots-20^{\circ} \mathrm{C}\right\rangle$ & RMSE & $\left\langle-1{ }^{\circ} \mathrm{C} \ldots-40^{\circ} \mathrm{C}\right\rangle$ & RMSE \\
\hline Levy-w & 0.042 & deVries-w & 0.045 & deVries-w & 0.049 & deVries-wi ${ }_{\mathrm{GMM}}$ & 0.057 \\
\hline Gori-vphf-w & 0.042 & deVries-wi GMM & 0.052 & Masch- $\perp-\mathrm{fib}_{\|}$ & 0.057 & Levy-wi $i_{\|}$ & 0.065 \\
\hline GeoMean-s $\mathrm{GMM}$ & 0.042 & Masch- $\perp-\mathrm{fib}_{\|}$ & 0.055 & Levy-wi $\mathbf{i}_{\|}$ & 0.059 & Levy-i-d $\|_{\|}$ & 0.068 \\
\hline deVries-w & 0.043 & Masch- $\perp$-fib & 0.058 & deVries-wi ${ }_{\mathrm{GMM}}$ & 0.060 & EMT-s ${ }_{\mathrm{GMM}}$ & 0.068 \\
\hline deVries-f & 0.045 & Maxwell-wi ${ }_{\mathrm{GMM}}$ & 0.059 & Levy-i-d ${ }_{\text {GMM }}$ & 0.064 & Masch-\|-fib ${ }_{\sum}$ & 0.069 \\
\hline EMT-s & 0.046 & EMT-s ${ }_{\mathrm{GMM}}$ & 0.062 & Gori-vphf-wi & 0.064 & Masch-\|-fib $b_{\mathrm{GMM}}$ & 0.069 \\
\hline Maxwell-w & 0.046 & Levy-i-d $\mathrm{d}_{\mathrm{GMM}}$ & 0.065 & Masch- $\perp-$ fib $_{\mathrm{GMM}}$ & 0.071 & deVries-w & 0.072 \\
\hline Levy-f-d $d_{\|}$ & 0.047 & Levy-wi ${ }_{\|}$ & 0.065 & Masch-\|-fib $\mathrm{GMM}$ & 0.072 & Gori-vphf-wi & 0.073 \\
\hline Masch- $\perp-$ fib $_{\mathrm{GMM}}$ & 0.051 & Gori-vphf-wi & 0.066 & EMT-S ${ }_{\mathrm{GMM}}$ & 0.072 & Maxwell-wi ${ }_{\mathrm{GMM}}$ & 0.074 \\
\hline Gori-hpi-w & 0.053 & Masch-\|-fib & 0.066 & deVries-i & 0.073 & Masch- $\perp-$ fib $_{\|}$ & 0.080 \\
\hline
\end{tabular}

$\lambda_{\text {wi }}$. Consequently, the $k_{j}$ must be evaluated individually for each meat having different initial water content. Evaluation of $\lambda_{\text {wi }}$ by the GMM model appears to be beneficial to deVries and Maxwell model, while the $\|$ model works better for Levy model. The use of $w$ as the continuous medium is beneficial only to deVries model, particularly from -1 to $-20{ }^{\circ} \mathrm{C}$. The use of $f a t$ as the continuous medium is beneficial only to foods with high fat content.

Overall, relatively small differences in predictions were observed between the best model versions by deVries, Levy, Mascheroni, Maxwell or Gori as applied to frozen meats with high water content. These differences could also be generated by uncertainty in food composition, temperature independent thermal conductivity of ice, measurement errors, and limitation of predictive models. The above results are preliminary and need to be confirmed by testing the models against the experimental data representing a wider variety of frozen foods. In addition, it would be beneficial to investigate the influence of temperature dependent thermal conductivity of all food components on the predictive model performance.

\section{Acknowledgements}

The authors wish to express their appreciation to Saint Mary's University in Canada, University of Rome-Tor Vergata, and Agenzia Spaziale Italiana for providing funds to complete this study. Acknowledgment is given to Mr Jeff Levy for his contribution to initial development of Excel simulations.

\section{References}

[1] A. Eucken, Allgemeine Gesetzmassigkeiten fur das Warmeleitvermogen verschiedener Stoffarten und Aggregatzustande, Forschung und Gebiete Ingenieur (Ausgabbe A) 11 (6) (1940).
[2] F.L. Levy, A modified Maxwell-Eucken equation for calculating the thermal conductivity of two-component solutions or mixtures, Int J Refrigeration 4 (1981) 223-225.

[3] M. Mattea, M.J. Urbicain, E. Rotstein, Prediction of thermal conductivity of vegetable foods by the effective medium theory, J Food Sci 51 (1) (1986) 113-115 [see also p. 134].

[4] R.H. Mascheroni, J. Ottino, A. Calvelo, A model for the thermal conductivity of frozen meat, Meat Sci 1 (1977) 235-243.

[5] Q.T. Pham, in: W.E.L. Spiess, H. Schubert (Eds.), Prediction of thermal conductivity of meats and other animal products from composition data, Engineering and Food vol. 1, Elsevier Applied Science, London, 1990, pp. 408-423.

[6] D.A. DeVries, Thermal properties of soils in: W.R. van Wijk (Ed.), Physics of plant environment, Wiley, New York, 1963, pp. 210-235.

[7] F. Gori, A theoretical model for predicting the effective thermal conductivity of unsaturated frozen soils, Proceedings of the fourth international conference on Permafrost, Fairbanks (Alaska), National Academy Press, Washington, DC, 1983. p. 363-368.

[8] J. Sundberg, Thermal properties of soils and rocks. Chalmers University of Technology and University of Gothenburg, Goteborg, Sweden. PhD Thesis; 1988, p. 1-310.

[9] K. Lichtenecker, Dielectric constant of natural and synthetic mixtures, Zeitschrift fur Physik 27 (1926) 115-158.

[10] O. Johansen, Thermal conductivity of soils. Trondheim, Norway, PhD Thesis; 1975 (CRREL translation 637, 1977).

[11] R. McGaw, Heat conduction in saturated granular materials. In: Effects of temperature and heat on engineering behavior of soils. Highway research board special report; 1969, vol. 103, p. $114-31$.

[12] H. Fricke, The electrical conductivity of a suspension of homogeneous spheroids, Phys Rev 24 (1924) 575.

[13] H.S. Carslaw, J.C. Jaeger, Conduction of heat in solids, 2nd ed, Oxford University Press, Oxford, 1986.

[14] D.A.G. Bruggeman, Dielectric constant and conductivity of mixtures of isotopic materials (in German), Ann Phys 24 (1935) 636-679.

[15] R. Landeauer, The electrical resistance of binary metallic mixtures, J Appl Phys 23 (1952) 779-784.

[16] D.J. Cleland, K.J. Valentas, Prediction of freezing time and design of food freezers in: R.P. Singh, K.J. Valentas (Eds.), Handbook of food engineering practice, CRC Press, Boca Raton, Florida, 1997, pp. 71-124. 
[17] Q.T. Pham, J. Willix, Thermal conductivity of fresh lamb meat, offals and fat in the range $-40-+30{ }^{\circ} \mathrm{C}$ : measurements and correlations, J Food Sci 54 (3) (1989) 508-515.

[18] Y. Choi, M.R. Okos, Effects of temperature and composition on the thermal properties of foods in: M. Le Maguer, P. Jelen
(Eds.), Food engineering and processes applications, transport phenomena, Elsevier Applied Science, Amsterdam, 1986, pp. 93-101.

[19] H.G. Schwartzberg, Effective heat capacity for the freezing and thawing of food, J Food Sci 41 (1976) 152-156. 